\title{
Addresses of Editors
}

Prof. Dr. Hans BungerT, Institut für Anglistik der Universität Regensburg, Universitätsstr. 31, D-8400 Regensburg 2

Prof. Dr. Hans-Jürgen DilleR, Englisches Seminar der Ruhr-Universität Bochum, Universitätsstr. 150, D-4630 Bochum 1

Prof. Dr. Herbert Grabes, Institut für Anglistik und Amerikanistik der Universität Gießen, Otto-Behaghel-Str. 10, D-6300 Gießen

\section{Addresses of Contributors}

Prof: Dr. Alfred Behrmann, Freie Universität Berlin, Fachbereich Germanistik, Habelschwerdter Allee 45, D-1000 Berlin 33

Marianne Brown, M.A., Office for Foreign Students, University of Oslo, Blindern, Oslo 3, Norway

Prof. Dr. Wilhelm G. Busse, Anglistisches Institut der Universität Düsseldorf, Universitätsstr. 1, D-4000 Düsseldorf 1

Carla Copenhaven, 1021 Verano Place, Irvine, CA 92715, U.S.A.

Prof. Dr. Vanna Gentrul, Università degli Studi di Roma, Facoltà di Magistero, Istituto di Lingua e Letteratura Inglese e di Letteratura Anglo-Americana, Via Magenta 2, I00185 Roma

Dr. Helen Hagenduchle, Im Schilf 3, CH-8044 Zürich

Prof. James L. Hitl, Dept. of English, Michigan State University, East Lansing, Michigan, U.S.A.

Prof. Dr. Lothar Honnighausen, Englisches Seminar, Universität Bonn, Regina-PacisWeg 5, D-5300 Bonn 1

Prof. Giorgio Melchiorı, Università degli Studi di Roma, Facoltà di Magistero, Via Magenta 2, I-00185 Roma

Dr. Luminitsa Niculescu, 4726 Elmwood Avenue, Los Angeles, CA 90004, U.S.A.

Prof. Dr. Kurt Tetzeli von Rosador, Englisches Seminar, Westfälische Wilhelms-Universität, Johannisstr. 12-20, D-4400 Münster

Dr. J. C. Whitehouse, The Modern Languages Centre, University of Bradford, Bradford, BD7 1DP, England 
